\title{
Estrogen Metabolic Process
}

National Cancer Institute

\section{Source}

National Cancer Institute. Estrogen Metabolic Process. NCI Thesaurus. Code C20136.

Estrogen Metabolism consists of diverse biochemical reactions primarily in the ovary that produce 19-carbon steroid hormones, which promote secondary female sexual development and menstrual function or convert such steroids to metabolic derivatives. 